\title{
Fonctions et feuilletages Levi-flat. Étude locale
}

\author{
DOMINIQUE CERVEAU - PAULO R. SAD
}

\begin{abstract}
We define the notion of CR equivalence for Levi-flat foliations and compare in a local setting these foliations to their linear parts. We study also the situation where the foliation has a first integral; a condition is given so that this integral is the real part of a holomorphic function.
\end{abstract}

Mathematics Subject Classification (2000): 32V10 (primary); 32V40, 37F75 (secondary).

\section{1. - Introduction}

Les hypersurfaces analytiques réelles Levi-flat de l'espace $\mathbb{C}^{n} \simeq \mathbb{R}^{2 n}$ ont été introduites et étudiées, tout de moins lorsque $n=2$, au début de $20^{\text {ème }}$ siècle par Almer et E. Cartan sous le nom d'hyperplanoïdes. On définit à l'époque un hyperplanoïde comme un lieu à un paramètre de surfaces caractéristiques ; on dirait aujourd'hui une hypersurface analytique réelle feuilletée en hypersurfaces complexes holomorphes.

Plus précisément soit $H \subset U \subset \mathbb{C}^{n}$ une hypersurface analytique réelle lisse d'un ouvert $U$ de $\mathbb{C}^{n}$. Le $\mathbb{R}$-hyperplan tangent $T_{x} H \subset T_{x} \mathbb{R}^{n}$ contient un unique espace vectoriel complexe de dimension maximale $n-1$, noté $T_{x}^{\mathbb{C}} H=$ $T_{x} H \cap i T_{x} H$ où $i=\sqrt{-1}$. On obtient ainsi un champ d'hyperplans complexes sur $H$; lorsque ce champ est intégrable au sens de Frobenius, l'hypersurface $H$ est feuilletée en hypersurfaces complexes, le feuilletage correspondant étant analytique réel. Dans cette éventualité on dit que $H$ est Levi-flat. Si $H \subset U \subset$ $\mathbb{C}^{n}$ est une hypersurface analytique réelle avec singularités nous dirons encore que $H$ est Levi-flat si sa partie lisse $H_{\text {lisse }}$ est elle même Levi-flat. Toutes ces notions se laissent évidemment germifier.

Le théorème suivant est dû à $\mathrm{E}$. Cartan [Car] : soit $H$ un germe d'hypersurface lisse analytique réel en l'origine de $\mathbb{C}^{n}$. Si $H$ est Levi-flat, il existe une submersion holomorphe $F$ telle que $H$ soit donné par $H=(\mathcal{R} e F=0)$. En particulier, $H$ peut être transformée biholomorphiquement en un hyperplan réel $\mathbb{R}^{2 n-1} \subset \mathbb{C}^{n}$. 
Disons à l'inverse, et sans trop entrer dans les détails, que $H$ est générique si le champ d'hyperplans $x \mapsto T_{x}^{\mathbb{C}}$ est lui même générique, en particulier non intégrable ; on peut penser à une condition plus forte du type $H$ ne contient pas de courbes holomorphes. La recherche d'une description qualitative des groupes d'automorphismes des domaines bornés a conduit maintes publications sur les surfaces géneriques. Citons le travail séminal de Chern et Moser [C-M] qui en donnent une forme normale holomorphe et les travaux récents de Baouendi et ses collaborateurs [Ba], où l'on trouvera les différentes problématiques sur le sujet.

Pour revenir aux hypersurfaces Levi-flat les premiers résultats significatifs concernant les cas singuliers sont dus à D. Burns et X. Gong en 1999 [B-G]. Ils démontrent qu'une quadrique réelle Levi-flat de $\mathbb{C}^{n}$ est de l'un des types suivants (à isomorphisme $\mathbb{C}$-linéaire près) :

1. $\mathcal{R e}\left(z_{1}^{2}+\cdots+z_{k}^{2}\right)=0, k \leq n$.

2. $\left(z_{1}+\bar{z}_{1}\right)\left(z_{2}+\bar{z}_{2}\right)=0$.

3. $z_{1} \bar{z}_{1}-z_{2} \bar{z}_{2}=0$.

4. $z_{1}^{2}+2 \lambda z_{1} \bar{z}_{1}+\bar{z}_{1}^{2}=0, \lambda \in \mathbb{R}$.

Ils établissent ensuite un résultat de type Morse pour les hypersurfaces Levi-flat ; en particulier si $H$ Levi-flat est donné en $0 \in \mathbb{C}^{n}$ par

$$
\mathcal{R} e\left(z_{1}^{2}+\cdots+z_{n}^{2}\right)+o\left(|z|^{2}\right)=0
$$

il existe un biholomorphisme qui transforme $H$ en la quadrique

$$
\mathcal{R} e\left(z_{1}^{2}+\cdots+z_{n}^{2}\right)=0 .
$$

Notre but ici est de présenter quelques propriétés élémentaires des feuilletages Levi-flat. Si $\mathcal{F}$ est un feuilletage analytique réel de codimension un (réelle) de l'ouvert $U \subset \mathbb{C}^{n}$, nous dirons que $\mathcal{F}$ est Levi-flat si les feuilles régulières de $\mathcal{F}$ le sont. Une telle notion peut, via les cartes, se globaliser et l'on a la notion de feuilletage analytique réel de codimension un (avec ou sans singularité) Levi-flat sur une variété complexe $M$. Nous commençons avec un résultat de rigidité dans l'esprit du point de vue adopté par les auteurs mentionnés ci-dessus : soit $P: \mathbb{C}^{n} \rightarrow \mathbb{R}$ un polynôme de degrée $N$. Supposons que le feuilletage défini par les niveaux de $P$ soit Levi-flat. Si P vérifie une certaine condition algébrique ouverte dans l'espace des polynômes de degré $N$, alors $P$ est la partie réelle d'un polynôme holomorphe.

Contrairement à cette situation, en général le feuilletage $\mathcal{F}^{\mathbb{C}}$ en feuilles holomorphes contenu dans un feuilletage Levi-flat $\mathcal{F}$ peut être non holomorphe (i.e. la structure transverse est non holomorphe). La notion naturelle d'équivalence est alors la suivante : deux feuilletages Levi-flat $\mathcal{F}_{1}$ et $\mathcal{F}_{2}$ définis sur les variétes complexes $M_{1}$ et $M_{2}$ sont CR-équivalents (ou CR conjugués) s'il existe $\Phi: M_{1} \rightarrow M_{2}$ un difféomorphisme analytique réel satisfaisant :

1. $\Phi$ conjugue $\mathcal{F}_{1}$ et $\mathcal{F}_{2}$.

2. $\Phi$ conjugue $\mathcal{F}_{1}^{\mathbb{C}}$ et $\mathcal{F}_{2}^{\mathbb{C}}$. 
3. $\Phi$ est holomorphe dans chaque feuille de $\mathcal{F}_{1}^{\mathbb{C}}$ à valeurs dans la feuille correspondante de $\mathcal{F}_{2}^{\mathbb{C}}$.

Cette notion va nous permettre de revisiter le théorème de Frobenius classique version Levi-flat : un feuilletage Levi-flat non singulier est localement CR conjugué au feuilletage donné par $\mathcal{R} e z_{1}=$ cste.

Ensuite nous étudions, du point de vue local, les feuilletages singuliers Levi-flat ayant un lieu singulier de type Kupka-Reeb. Selon la position du lieu singulier par rapport au feuilletage par hypersurfaces complexes (tangence ou transversalité), nous pouvons les comparer aux feuilletages linéarisés et dans certains cas prouver que l'équivalence CR est effectivement holomorphe (Théorèmes 6.1 et 8.2).

Nous démontrons aussi (Théorème 7.1) un "lemme de Morse" Levi-flat en dimension supérieure à trois.

\section{2. - Notations-définitions et exemples}

Nous allons préciser les notions précédentes en adoptant délibérément un point de vue à la Cartan. On munit $\mathbb{C}^{n}$ des coordonnées $\left(z_{1}, \ldots, z_{n}\right), z_{j}=$ $x_{j}+y_{j}$. Soit $\mathcal{F}$ un feuilletage analytique réel (éventuellement singulier) de codimension un sur l'ouvert $U$ de $\mathbb{C}^{n}$ défini par la 1-forme intégrable analytique réelle

$$
\omega_{1}=\sum_{j} a_{j} d x_{j}+\sum_{j} b_{j} d y_{j}, \quad a_{j}, b_{j} \in \mathcal{O}_{\mathbb{R}}(U) .
$$

On peut, quitte à diviser $\omega_{1}$ par le pgcd des $a_{j}, b_{j}$, supposer que $\omega_{1}$ ne s'annulent que sur un ensemble $\operatorname{Sing}(\mathcal{F})$ de codimension deux réelle.

On associe à chaque point $z \in U$ régulier du feuilletage $\mathcal{F}$ l'hyperplan complexe $\Sigma_{z}^{\mathbb{C}}(\mathcal{F})$ tangent à la feuille par $z$; le champ d'hyperplans $\Sigma^{\mathbb{C}}(\mathcal{F})$ est donné par le noyau de la 1 -forme différentielle analytique (à valeurs complexes)

$$
\Omega=\sum_{j}\left(a_{j}-i b_{j}\right) d z_{j}
$$

cette 1-forme se prolonge naturellement (par la formule) à $U$ (et ne s'annule que sur un ensemble de codimension deux réelle). Le champ $\Sigma^{\mathbb{C}}(\mathcal{F})$ est analytique réel, mais pas forcément holomorphe.

L'égalité $\Omega=\omega_{1}+i \omega_{2}$ avec

$$
\omega_{2}=-\sum_{j} b_{j} d x_{j}+\sum_{j} a_{j} d y_{j}
$$

entraine que $\Sigma^{\mathbb{C}}(\mathcal{F})$ est aussi défini par le système de Pfaff $\left\langle\omega_{1}, \omega_{2}\right\rangle$. 
Le feuilletage $\mathcal{F}$ est Levi-flat si $\Sigma^{\mathbb{C}}(\mathcal{F})$ est intégrable ; dans cette situation, on obtient un feuilletage $\mathcal{F}^{\mathbb{C}}$ dont les feuilles sont holomorphes.

Lorsque $\omega_{1}=d f$, où $f: U \subset \mathbb{C}^{n} \rightarrow \mathbb{R}$ est une fonction analytique réelle sur l'ouvert $U$ de $\mathbb{C}^{n}$, le champ d'hyperplans complexes $\Sigma^{\mathbb{C}}(f)$ est défini par

$$
\Omega=\frac{\partial f}{\partial z} d z=\sum_{i=1}^{n} \frac{\partial f}{\partial z_{i}} d z_{i}
$$

et les 1-formes différentielles analytiques $\omega_{1}$ et $\omega_{2}$ sont donnés par

$$
\begin{aligned}
& \omega_{1}=d_{\mathbb{R}} f=\sum_{j} \frac{\partial f}{\partial x_{j}} d x_{j}+\sum_{j} \frac{\partial f}{\partial y_{j}} d y_{j} \\
& \omega_{2}=-\sum_{j} \frac{\partial f}{\partial y_{j}} d x_{j}+\sum_{j} \frac{\partial f}{\partial x_{j}} d y_{j},
\end{aligned}
$$

La fonction $f$ est Levi flat si $\Sigma^{\mathbb{C}}(f)$ est intégrable.

Dans le cas particulier où $f$ est la partie réelle d'une fonction holomorphe $F=f+i g$, on observe que $d \omega_{2}=0$ ce qui est que la traduction des conditions de Cauchy-Riemann :

$$
\omega_{2}=-\sum_{j} \frac{\partial f}{\partial y_{j}} d x_{j}+\sum_{j} \frac{\partial f}{\partial x_{j}} d y_{j}=\sum_{j} \frac{\partial g}{\partial x_{j}} d x_{j}+\sum_{j} \frac{\partial g}{\partial y_{j}} d y_{j} .
$$

Évidemment ici $\Sigma^{\mathbb{C}}(f)$ est holomorphe et définit un feuilletage holomorphe régulier sur l'ouvert $U$ privé d'un ensemble analytique (ici complexe) de codimension $\leq 2$; les feuilles de ce feuilletage sont bien sûr les niveaux de $F$.

Toutes les définitions présentées sont invariantes par biholomorphismes et s'implantent sur une variété complexe.

Comme nous l'avons déjà dit, le champ d'hyperplans $\Sigma^{\mathbb{C}}(f)$ n'est pas intégrable ni holomorphe en géneral. Examinons pour constater celà quelques exemples. Considérons sur $\mathbb{C}^{n}$ la forme quadratique associée au produit hermitien standart $Q(z)=\sum_{j}\left|z_{j}^{2}\right|=\sum_{j}\left(x_{j}^{2}+y_{j}^{2}\right)$. Alors $\Sigma^{\mathbb{C}}(Q)$ ne peut pas être intégrable, sinon une feuille générique serait l'image par une immersion d'une variété complexe de dimension $n-1$ contenue dans une fibre de $Q$ et donc bornée, ce qui est absurde par le principe du maximum (en fait, un calcul explicite donne $\Omega=\frac{\partial Q}{\partial z} d z=2 \sum_{j} \bar{z}_{j} d z_{j}$, et on constate via cette formule que le champ d'hyperplans $\Sigma^{\mathbb{C}}(Q)$ n'est pas holomorphe, pas plus qu'intégrable).

Donnons maintenant l'exemple d'une fonction analytique réelle $f: U \rightarrow \mathbb{R}$ tel que $\Sigma^{\mathbb{C}}(f)$ soit intégrable, mais ne définisse pas un feuilletage holomorphe (cet exemple nous a été indiqué par J-M. Lion). Pour celà, considérons une application $A: \mathbb{C} \rightarrow \mathbb{C}, \mathbb{R}$-linéaire inversible mais non $\mathbb{C}$-linéaire et faisons la construction suivante dans $\mathbb{C}^{2}$. En joignant les points $\left(0, z_{2}\right)$ et $\left(1, A z_{2}\right)$ par une droite complexe nous construisons un feuilletage $\mathcal{G}$ analytique réel de 
codimension 2 au voisinage de l'origine dans $\mathbb{C}^{2}$. C'est un feuilletage en feuilles holomorphes, non singulier en $(0,0) \in \mathbb{C}^{2}$ qui ne peut être holomorphe, sinon $A$ serait $\mathbb{C}$-linéaire. Par le théorème de Frobenius ordinaire, il existe des fonctions analytiques réelles $f$ et $g$ à l'origine de $\mathbb{C}^{2}$, intégrales premières de $\mathcal{G}$. Le feuilletage non singulier donné au voisinage de $(0,0) \in \mathbb{C}^{2}$ par les fibres de $f$ est Levi-flat et le champ d'hyperplans $\Sigma^{\mathbb{C}}(f)$ n'est pas holomorphe (il est holomorphe ssi $A$ est $\mathbb{C}$-linéaire). En fait, on peut choisir les fonctions $f$ et $g$ de la façon suivante : donné $(x, y) \in \mathbb{C}^{2}$ au voisinage de l'origine, il existe une droite par les points $(x, y),\left(0, z_{2}\right)$ et $\left(1, A z_{2}\right)$; évidenment $z_{2}$ s'écrit comme une fonction rationelle de dégree 1 des coordonnées réelles de $x$ et $y$. On prend $f$ et $g$ comme $\mathcal{R} e z_{2}$ et $\mathcal{I} m z_{2}$.

\section{3. - Un résultat de rigidité}

Nous nous plaçons dans cette section dans un contexte algébrique global. Introduisons dans un premier temps une condition algébrique de non dégénérescence pour un feuilletage Levi-flat donné par les niveaux d'une fonction analytique $f:\left(\mathbb{C}^{n}, 0\right) \rightarrow(\mathbb{R}, 0)$ non submersive en $0 \in \mathbb{C}^{n}$. Soient $\omega_{1}$ et $\omega_{2}$ définies par (2.4). Comme ces 1-formes différentielles sont analytiques réelles à l'origine de $\mathbb{R}^{2 n} \simeq \mathbb{C}^{n}$, on peut considérer leur complexifiés $\omega_{1}^{\mathbb{C}}$ et $\omega_{2}^{\mathbb{C}}$ obtenus par substitution de variables complexes $u_{j}$ et $v_{j}$ en place de $x_{j}$ et $y_{j}$; les 1-formes différentielles $\omega_{1}^{\mathbb{C}}$ et $\omega_{2}^{\mathbb{C}}$ sont holomorphes à l'origine de $\mathbb{C}^{2 n}$ et bien sûr, de par l'hypothèse Levi-flat, définissent un feuilletage de codimension 2 complexe en l'origine de $\mathbb{C}^{n}$. Le lieu singulier $\operatorname{Sing}\left(\omega_{1}^{\mathbb{C}} \wedge \omega_{2}^{\mathbb{C}}\right)$ de la 2-forme $\omega_{1}^{\mathbb{C}} \wedge \omega_{2}^{\mathbb{C}}$ est l'ensemble analytique complexe

$$
\operatorname{Sing}\left(\omega_{1}^{\mathbb{C}} \wedge \omega_{2}^{\mathbb{C}}\right)=\left\{m \in \mathbb{C}^{2 n} ;\left(\omega_{1}^{\mathbb{C}} \wedge \omega_{2}^{\mathbb{C}}\right)(m)=0\right\}
$$

Nous dirons que $f$ est non dégénérée si $\operatorname{cod} \operatorname{Sing}\left(\omega_{1}^{\mathbb{C}} \wedge \omega_{2}^{\mathbb{C}}\right) \geq 3$.

EXEMPLE 3.1. Soit $f=\mathcal{R} e\left(z_{1}^{2}+\cdots+z_{2}^{2}\right)$; un calcul élémentaire montre que $f$ est non dégénérée pour $n \geq 3$, mais ne l'est pas pour $n=2$. En fait, supposons, avec les notations précédents, que $m \in \operatorname{Sing}\left(\omega_{1}^{\mathbb{C}} \wedge \omega_{2}^{\mathbb{C}}\right)$ et $\omega_{1}^{\mathbb{C}}(m) \neq 0$. Alors en $m$ on a $\omega_{2}^{\mathbb{C}}=\lambda \omega_{1}^{\mathbb{C}}(m)$ pour un certain $\lambda \in \mathbb{C}$; si l'on écrit explicitement $\left(f^{\mathbb{C}}\right.$ est le complexifié de $f$ ) on obtient pour $j=1, \ldots, n$ :

$$
\begin{aligned}
& \frac{\partial f^{\mathbb{C}}}{\partial u_{j}}(m)=-\lambda \frac{\partial f^{\mathbb{C}}}{\partial v_{j}}(m) \\
& \frac{\partial f^{\mathbb{C}}}{\partial v_{j}}(m)=\lambda \frac{\partial f^{\mathbb{C}}}{\partial u_{j}}(m),
\end{aligned}
$$

et l'on constate que $\lambda= \pm i$. Par suite $\operatorname{Sing}\left(\omega_{1}^{\mathbb{C}} \wedge \omega_{2}^{\mathbb{C}}\right)$ contient l'ensemble $\left\{m \in \mathbb{C}^{2} ; \omega_{2}^{\mathbb{C}}(m)= \pm i \omega_{1}^{\mathbb{C}}(m)\right\}$, et cet ensemble est pour $n=2$ de codimension 
2 (ou vide, mais c'est le cas submersif). Ainsi la notion de non dégénérescence n'est opérante qu'en dimension $n \geq 3$.

On a le théorème suivant à la Malgrange :

ThÉORÈme 3.1. Soit $f:\left(\mathbb{C}^{n}, 0\right) \rightarrow(\mathbb{R}, 0)$ analytique réelle Levi-flat. Si $f$ est non dégénérée, il existe $g:\left(\mathbb{C}^{n}, 0\right) \rightarrow(\mathbb{R}, 0)$ analytique réelle intégrale première de $\Sigma^{\mathbb{C}}(f)$ telle que $d f \wedge d g \neq 0$. En particulier, les feuilles locales de $\Sigma^{\mathbb{C}}(f)$ sont les composantes connexes des niveaux de $f+i \mathrm{~g}$, et sont donc des hypersurfaces holomorphes fermées (dans un petit ouvert où les objets sont représentés).

Preuve. Le système de Pfaff $<\omega_{1}^{\mathbb{C}}, \omega_{2}^{\mathbb{C}}>$ à l'origine de $\mathbb{C}^{2 n}$ est intégrable et satisfait les hypothèses du Frobenius II de Malgrange [Mal II]. Comme on sait déjà que $\omega_{1}^{\mathbb{C}}=d f^{\mathbb{C}}$, ceci produit une fonction holomorphe $\tilde{g}:\left(\mathbb{C}^{n}, 0\right) \rightarrow(\mathbb{C}, 0)$ telle que $<\omega_{1}^{\mathbb{C}}, \omega_{2}^{\mathbb{C}}>=<d f^{\mathbb{C}}, d \tilde{g}>$. Comme le système est construit par complexification d'un système réel on peut supposer que $\tilde{g}=g^{\mathbb{C}}$ pour une certaine fonction analytique $g:\left(\mathbb{C}^{n}, 0\right) \rightarrow(\mathbb{R}, 0)$. D'où le résultat.

En appliquant le Frobenius I de Malgrange [Mal I], on obtient la généralisation suivante. Soit $\mathcal{F}$ un feuilletage analytique réel Levi-flat en $0 \in \mathbb{C}^{n}$ donné par $\omega_{1}$ intégrable; si $\omega_{2}$ est comme dans (2.3), on dit encore que $\mathcal{F}$ est non dégénérée en $0 \in \mathbb{C}^{n}$ si codSing $\left(\omega_{1}^{\mathbb{C}} \wedge \omega_{2}^{\mathbb{C}}\right) \geq 3$.

ThÉoRème 3.2. Soit $\mathcal{F}$ feuilletage Levi-flat non dégénérée en $0 \in \mathbb{C}^{n}$. Il existe deux fonctions analytiques réelles $f$ et $g$ satisfaisant :

1. f est intégrale première de $\mathcal{F}$, plus précisement, $\omega_{1}=a d f$, où a est une unité.

2. $d f \wedge d g \not \equiv 0$ et $g$ est intégrale première de $\Sigma^{\mathbb{C}}(\mathcal{F})$, plus précisement $<$ $\omega_{1}, \omega_{2}>=<d f, d g>$.

Preuve. D'après Frobenius I, il existe $f$ satisfaisant 1 ) ; on applique alors le Théorème 3.1 à cet $f$.

Venons en maintenant au résultat annoncé dans l'introduction. Soit $P$ : $\mathbb{C}^{n} \rightarrow \mathbb{R}$ un polynôme réel que nous écrivons sous la forme $P=P_{0}+\cdots+P_{N}$, où chaque $P_{k}$ est homogène de degré $k$

ThÉorème 3.3. Supposons que le polynôme $P$ soit Levi-flat. Si $P_{N}$ est non dégénéré, il existe un polynôme complexe $P^{\mathbb{C}}: \mathbb{C}^{n} \rightarrow \mathbb{C}$ tel que $P=\mathcal{R e} P^{\mathbb{C}}$. En particulier le feuilletage $\Sigma^{\mathbb{C}}(P)$ est holomorphe, en fait donné par les niveaux de $P^{\mathbb{C}}$.

Preuve. Elle s'appuie sur un argument de division utilisé par Malgrange dans les preuves de Frobenius I et II. Rappelons le Lemme de Division de deRham-Saito [Sa] :

LeMme. Soient $\alpha_{1}, \cdots, \alpha_{k}$ des germes de 1-formes holomorphes à l'origine de $\mathbb{C}^{n} ;$ supposons que cod $\operatorname{Sing}\left(\alpha_{1} \wedge \cdots \wedge \alpha_{k}\right) \geq p+1$ où $p \in \mathbb{N}$. Alors sont équivalents pour $\Theta$ p-forme holomorphe à l'origine de $\mathbb{C}^{n}$ :

1. $\Theta \wedge \alpha_{1} \wedge \cdots \wedge \alpha_{k}=0$

2. $\Theta$ s'écrit $\Theta=\sum_{j} \beta_{j} \wedge \alpha_{j}$, où les $\beta_{j}$ sont des $p-1$ formes holomorphes. 
Nous appliquons ce résultat pour $p=2$, i.e., pour les 2-formes différentielles.

Considérons comme d'habitude les 1-formes différentielles $\omega_{1}=d P=$ $d P_{1}+\cdots+d P_{N}$ et $\omega_{2}=\omega_{2}^{0}+\cdots+\omega_{2}^{N-1}$, où chaque $\omega_{2}^{k}$ est une 1 -forme différentielle à coefficients homogènes de dégré $k$. Nous allons montrer que $\omega_{2}$ est fermée, i.e. que chaque $\omega_{2}^{k}$ est fermée. La condition Levi-flat dit que

$$
\omega_{1} \wedge \omega_{2} \wedge d \omega_{2}=0
$$

égalité que l'on peut complexifier. On peut alors appliquer le Lemme de Division (aux complexifés) puisque $P_{N}$ est non dégénérée :

$$
d \omega_{2}^{N-1, \mathbb{C}}=\beta_{1} \wedge d P_{N}+\beta_{2} \wedge \omega_{2}^{N-1, \mathbb{C}},
$$

où les 1 -formes différentielles $\beta_{j}$ peuvent être choisies à coefficients homogènes. Mais les coefficients de $d \omega_{2}^{N-1}$ étant de degré plus petit que ceux de $\omega_{2}^{N-1}$ et $d P_{N}$, ces $\beta_{j}$ sont donc nuls et $d \omega_{2}^{N-1}=0$. alors

Supposons que l'on ait montré que $d \omega_{2}^{j}=0$ pour $k<j \leq N-1$. On a

$$
d\left(P_{0}+P_{1}+\cdots P_{N}\right) \wedge\left(\omega_{2}^{0}+\cdots+\omega_{2}^{N-1}\right) \wedge d\left(\omega_{2}^{0}+\cdots+\omega_{2}^{k}\right)
$$

dont le terme de plus haut degré est

$$
d P_{N} \wedge \omega_{2}^{N-1} \wedge d \omega_{2}^{k}=0 .
$$

Le même argument que précédemment dit que $d \omega_{2}^{k}=0$; ainsi $\omega_{2}$ est fermée et il existe donc un polynôme $Q$ tel que $\omega_{2}=d Q$.

Comme nous l'avons déjà vu, la condition $\omega_{2}$ fermée donne les conditions de Cauchy-Riemann, c'est à dire que $P^{\mathbb{C}}=P+i Q$ est holomorphe.

Il n'exist pas un résultat comme le Théorème 3.3 dans le cadre rationel ; par exemple, si l'on regarde la construction à la fin de la Section 2, en dégree 1 le feuilletage par droites n'est pas génériquement holomorphe (parce que ceci dépend d'un choix $\mathbb{C}$-linéaire de $A$ ).

\section{4. - Frobenius non singulier Levi-flat}

Nous nous proposons d'établir le

THÉORÈME 4.1. Soit $\mathcal{F}$ un feuilletage analytique réel Levi-flat non singulier à l'origine de $\mathbb{C}^{n}$, de codimension un réelle. Alors $\mathcal{F}$ est $C R$-conjugué au feuilletage associé à la fonction $\mathcal{R e} z_{n}$. 
Preuve. Par le théorème de Frobenius classique, il existe une submersion locale $f$ en $0 \in \mathbb{C}^{n}$ intégrale première de $\mathcal{F}$; quitte à faire un changement de coordonnées $\mathbb{C}$-linéaire, on peut supposer que la différentielle de $f$ en $0 \in \mathbb{C}^{n}$ coïncide avec $d x_{n}=d \mathcal{R} e z_{n}$. Par suite, la feuille de $\mathcal{F}^{\mathbb{C}}$ passant par 0 est tangente à l'hyperplan $z_{n}=0$. En résulte que les feuilles voisines de $\Sigma^{\mathbb{C}} \mathcal{F}$ sont transverses à la fibration verticale $\left(z_{1}, \ldots, z_{n-1}\right)=$ cste ; ainsi la feuille passant par le point $(0, \ldots, 0, t) \in \mathbb{C}^{n}$ est le graphe d'une fonction holomorphe $\left(z_{1}, \ldots, z_{n-1}\right) \mapsto \phi_{t}\left(z_{1}, \ldots, z_{n-1}\right)$ dépendant $\mathbb{R}$-analytiquement du paramètre $t \in \mathbb{C}$.

Considérons l'application analytique réelle $\Psi:\left(\mathbb{C}^{n}, 0\right) \rightarrow\left(\mathbb{C}^{n}, 0\right)$ définie $\operatorname{par}\left(z_{1}, \ldots, z_{n-1}, z_{n}\right) \mapsto\left(z_{1}, \ldots, z_{n-1}, \phi_{z_{n}}\left(z_{1}, \ldots, z_{n-1}\right)\right)$. Comme $\phi(0)=z_{n}$, $\Psi$ est un difféomorphisme local et, à $z_{n}$ fixé, $\Psi$ est holomorphe. On constate facilement que $\Psi$ est une conjugaison CR entre le feuilletage holomorphe $z_{n}=$ cste et le feuilletage $\Sigma^{\mathbb{C}} \mathcal{F}$.

L'application $f \circ \Psi$ est intégrale première de $z_{n}=$ cste ; dit autrement, $f \circ$ $\Psi(z)=l\left(z_{n}, \bar{z}_{n}\right)$ pour une certaine fonction analytique réelle $l$ définie à l'origine de $\mathbb{C}$. Visiblement, $l$ est submersive et donc il existe un difféo- morphisme analytique $h:(\mathbb{C}, 0) \rightarrow(\mathbb{C}, 0)$ tel que $l \circ h\left(z_{n}\right)=\mathcal{R} e z_{n}$. Finalement l'application $\tilde{\Psi}:\left(z_{1}, \ldots, z_{n-1}, z_{n}\right) \mapsto\left(z_{1}, \ldots, z_{n-1}, \phi_{h\left(z_{n}\right)}\left(z_{1}, \ldots, z_{n-1}\right)\right)$ satisfait $f \circ \tilde{\Psi}=$ $\mathcal{R}\left(z_{n}\right)$ et donne une conjugaison $\mathrm{CR}$ entre le feuilletage $\mathcal{R} e z_{n}=$ cste et $\mathcal{F}$.

\section{5. - Feuilletages linéaires}

Disons qu'un feuilletage sur $\mathbb{R}^{m}$ est linéaire s'il est donné par une 1-forme différentielle intégrable $\eta=\sum_{j} c_{j} d x_{j}$ où les $c_{j}$ sont des fonctions linéaires qui ne s'annulent pas sur un même hyperplan réel.

G. Reeb $[\mathrm{Re}]$ a établi le résultat suivant (valable aussi dans le cas complexe) :

THÉORÈME. Soit $\eta$ comme ci-dessus ; on a l'alternative suivante :

1. $\eta$ est fermée, i.e., $\eta=d Q$ où $Q$ est quadratique.

2. il existe un système de coordonnées linéaires $u_{1}, \ldots, u_{n}$ de $\mathbb{R}^{m}$ dans lequel $\eta$ s'écrit

$$
\eta=\left(\alpha_{1} u_{1}+\alpha_{2} u_{2}\right) d u_{1}+\left(\beta_{1} u_{1}+\beta_{2} u_{2}\right) d u_{2}
$$

où les $\alpha_{j}, \beta_{j}$ sont des constantes. Dit autrement, $\eta$ ne dépend que de deux variables.

En voici la variante Levi-flat :

THÉORÈME 5.1. Soit $\mathcal{F}$ un feuilletage (réel) linéaire de $\mathbb{C}^{n} \simeq \mathbb{R}^{2 n}$ donné par une 1-forme différentielle intégrable $\omega$. Si $\mathcal{F}$ est Levi-flat, alors $\omega$ est de l'un des 
types suivants (dans un système de coordonnées linéaires complexes $z_{1} \cdots, z_{n}$ ):

$$
\begin{aligned}
& \omega=d\left(\mathcal{R} e\left(z_{1}^{2}+\ldots z_{k}^{2}\right)\right), k \leq n \\
& \omega=x_{1} d x_{2}-x_{2} d x_{1}, \quad x_{j}=\mathcal{R} e z_{j}, j=1,2 \\
& \omega=\left(\alpha_{1} x_{1}+\alpha_{2} y_{1}\right) d x_{1}+\left(\beta_{1} x_{1}+\beta_{2} y_{1}\right) d y_{1}, \quad z_{1}=x_{1}+i y_{1} .
\end{aligned}
$$

Preuve. On utilise le théorème de Reeb. Lorsque $\omega=d Q$, le niveau $Q=0$ est Levi-flat singulier. On peut alors invoquer le résultat de $[B-G]$ qui dit que $Q$ est de l'un des types suivantes :

$$
\begin{array}{ll}
\text { a) } & \operatorname{Re}\left(z_{1}^{2}+\ldots z_{k}^{2}\right), k \leq n . \\
\text { b) } & \left(z_{1}+\bar{z}_{2}\right)\left(z_{2}+\bar{z}_{2}\right)=4 x_{1} x_{2} . \\
\text { c) } & z_{1} \bar{z}_{1}-z_{2} \bar{z}_{2}=x_{1}^{2}+y_{1}^{2}-x_{2}^{2}-y_{2}^{2} . \\
\text { d) } & z_{1}^{2}+2 \lambda z_{1} \bar{z}_{1}+\bar{z}_{1}^{2}, \lambda \in \mathbb{R} .
\end{array}
$$

Les cas a) et d) entrent dans notre énoncé. Dans les cas b) et c), un calcul élémentaire montre que $\Sigma^{\mathbb{C}} \mathcal{F}$ n'est pas intégrable.

Plaçons nous maintenant dans la deuxième alternative de Reeb, où $\omega=$ $\left(\alpha_{1} u_{1}+\alpha_{2} u_{2}\right) d u_{1}+\left(\beta_{1} u_{1}+\beta_{2} u_{2}\right) d u_{2}$. Si le 2-plan réel défini par les coordonnées $\left\{u_{1}, u_{2}\right\}$ est complexe, après un changement $\mathbb{R}$-linéaire de coordonnées on se trouve dans l'éventualité 3 ). Sinon, on écrit $u_{1}=\mathcal{R} e z_{1}$ et $u_{2}=\mathcal{R} e z_{2}$ où $z_{1}$ et $z_{2}$ sont des coordonnées complexes indépendantes. On note $z_{j}=x_{j}+i y_{j}$, et ainsi (selon (2.1) et (2.3)) :

$$
\begin{aligned}
& \omega_{1}=\left(\alpha_{1} x_{1}+\alpha_{2} x_{2}\right) d x_{1}+\left(\beta_{1} x_{1}+\beta_{2} x_{2}\right) d x_{2} \\
& \omega_{2}=\left(\alpha_{1} x_{1}+\alpha_{2} x_{2}\right) d y_{1}+\left(\beta_{1} x_{1}+\beta_{2} x_{2}\right) d y_{2}
\end{aligned}
$$

On a :

$\omega_{1} \wedge \omega_{2} \wedge d \omega_{2}=\left(\alpha_{2} \beta_{1}-\beta_{2} \alpha_{1}\right)\left(\alpha_{1} x_{1}^{2}+\left(\alpha_{2}+\beta_{1}\right) x_{1} x_{2}+\beta_{2} x_{2}^{2}\right) d x_{1} \wedge d x_{2} \wedge d y_{1} \wedge d y_{2}$.

Puisque $\omega_{1}$ ne s'annule pas sur un hyperplan (i.e. $\alpha_{2} \beta_{1}-\beta_{2} \alpha_{1} \neq 0$ ), on voit que $\omega_{1} \wedge \omega_{2} \wedge d \omega_{2}=0$ si et seulement si $\alpha_{1}=\beta_{2}=0$ et $\alpha_{2}+\beta_{1}=0$. D'où le résultat.

Remarque. Dans le premier cas, le feuilletage $\Sigma^{\mathbb{C}} \mathcal{F}$ est donné par les niveaux de la forme quadratique $z_{1}^{2}+\cdots+z_{k}^{2}$; dans le troisième, $\Sigma^{\mathbb{C}} \mathcal{F}$ correspond aux niveaux de $z_{1}$. Dans ces deux cas $\Sigma^{\mathbb{C}} \mathcal{F}$ est holomorphe. Par contre, dans l'éventualité 2), $\Sigma^{\mathbb{C}} \mathcal{F}$ n'est pas holomorphe parce qu'il est défini par $\Omega=x_{2} d z_{1}-x_{1} d z_{2}$.

Dans les sections suivantes nous étudions un certain nombre de feuilletages locaux Levi-flat dont les parties linéaires sont donnés par le Théorème 5.1. 


\section{6. - Phénomène de Kupka-Reeb avec ensemble singulier holomorphe}

On analyse dans cette section les perturbations non-linéaires intégrables et Levi-flat de la 1 -forme linéaire donné par (5.3). Lorsque $\alpha_{2} \neq \beta_{1}$ nous sommes en présence d'un phénomène de Kupka-Reeb. Dans le cas classique, ce phénomène est décrit par l'énoncé suivant, du à $\mathrm{I}$. Kupka $[\mathrm{K}]$, conséquence du théorème de Darboux modélisant les 2-formes fermées :

THÉORÈME. Soit $\eta$ un germe de 1-forme intégrable analytique réelle à l'origine de $\mathbb{R}^{m}$. On suppose que $\eta(0)=0$ et $d \eta(0) \neq 0$. Il existe un système de coordonnées $u_{1}, \ldots u_{n}$ de $\mathbb{R}^{m}$ dans lequel $\eta$ s'écrit

$$
\eta=a\left(u_{1}, u_{2}\right) d u_{1}+b\left(u_{1}, u_{2}\right) d u_{2} .
$$

Une façon équivalente de le dire est la suivante : si $\mathcal{G}$ est le feuilletage associé à $\eta, \mathcal{G}$ apparait comme pull-back via la projection $\left(u_{1}, \ldots, u_{m}\right) \mapsto$ $\left(u_{1}, u_{2}\right)$ d'un feuilletage $\mathcal{G}_{0}$ en dimension 2. On note parfois $\mathcal{G} \simeq G_{0} \times \mathbb{R}^{m-2}$. L'ensemble singulier est évidemment $u_{1}=u_{2}=0$; on dit qu'il est de type Kupka-Reeb.

Le théorème ci dessus est aussi valable dans le cadre holomorphe, en classe $C^{\infty}$ et même en classe $C^{r}, r \geq 1$, mais nous n'en ferons pas usage.

Soit $\mathcal{F}$ un germe de feuilletage Levi-flat à l'origine de $\mathbb{C}^{n}$ défini par la 1-forme différentielle $\omega$ avec partie linéaire (5.3) :

$$
\omega_{\text {lin }}=\left(\alpha_{1} x_{1}+\alpha_{2} y_{1}\right) d x_{1}+\left(\beta_{1} x_{1}+\beta_{2} y_{1}\right) d y_{1}, \quad z_{1}=x_{1}+i y_{1} .
$$

tel que

1. $d \omega(0) \neq 0$.

2. la matrice

$$
\left(\begin{array}{cc}
-\beta_{1} & -\beta_{2} \\
\alpha_{1} & \alpha_{2}
\end{array}\right)
$$

est $\mathbb{C}$-diagonalisable, les valeurs propres $\lambda$ et $\mu$ sont différents de 0 et le quotient $\frac{\lambda}{\mu}$ n'est pas réel negatif.

3. $\frac{\lambda}{\mu}, \frac{\mu}{\lambda} \notin \mathbb{N}^{*} \backslash\{1\}$ (sans résonances).

Soit $\mathcal{F}_{\text {lin }}$ le feuilletage linéaire donné par $\omega_{\text {lin }}=0$. Les feuilles de $\mathcal{F}_{\text {lin }}^{\mathbb{C}}$ sont données par $z_{1}=$ cste, donc $\mathcal{F}_{\text {lin }}^{\mathbb{C}}$ est holomorphe et s'étend en un feuilletage sans singularités le long de $\left\{z_{1}=0\right\}$ qui est une singularité "apparente". Notre but dans cette section est de démontrer le

THÉORÈME 6.1. Il existe une conjugaison continue entre $\mathcal{F}$ et $\mathcal{F}_{\text {lin }}$; cette conjugaison est $C R$ en dehors des ensembles singuliers. 
Preuve. Le premier fait qu'il faut remarquer d'après la nature de $\omega_{\text {lin }}$ est que l'espace tangent en chaque point de l'ensemble singulier est un hyperplan complexe ; donc l'ensemble singulier est lui même une sousvariété lisse complexe de codimension (complexe) un. On peut alors supposer qu'il est donné par $z_{1}=0$.

Pour simplifier l'exposé, on suppose $n=2$ (de façon équivalente, on coupe le feuilletage par les 2-plans complexes contenant l'axe des $z_{1}$ ). D'après le théorème de Kupka (voir condition (1)), il existe $\phi$, un difféomorphisme local en $0 \in \mathbb{C}^{2}$, réel analytique, tel que $\phi^{*} \omega=A d z_{1}+\bar{A} d \bar{z}_{1}$, où $A$ est une fonction analytique réelle satisfaisant $A(0)=0$ (car $z_{1}=0$ est l'ensemble singulier) donc $A\left(z_{1}, \bar{z}_{1}\right)=\alpha z_{1}+\beta \bar{z}_{1}, \alpha$ et $\beta$ fonctions analytiques réelles ([B-G], Lemma 3.8). Écrivons $\phi\left(z_{1}, z_{2}\right)=\left(a z_{1}+b \bar{z}_{1}, \gamma\right)$, où $a, b$ et $\gamma$ sont aussi des fonctions analytiques réelles (la première composant de $\phi$ est nulle sur l'ensemble $\left\{z_{1}=0\right\}$ puisque cet ensemble est invariant par $\phi)$. Comme $\omega=\left(\phi^{-1}\right)^{*}\left(\mathcal{R} e A d z_{1}\right)$, on trouve d'après un calcul élémentaire :

$$
\omega=\operatorname{Re}\left(A^{\prime} d z_{1}+B^{\prime} d z_{2}\right)
$$

avec $A^{\prime} \in<z_{1}, \bar{z}_{1}>$ et $B^{\prime} \in<z_{1}^{2}, \bar{z}_{1}^{2}, z_{1} \bar{z}_{1}>$. Le feuilletage $\mathcal{F}^{\mathbb{C}}$ est donné par $\Omega=A^{\prime} d z_{1}+B^{\prime} d z_{2}=0$.

Affirmation. il existe $c \in \mathbb{R}$, positif, tel que $\left|\frac{B^{\prime}}{A^{\prime}}\right| \leq c\left|z_{1}\right|$. En fait, $\left|\frac{B^{\prime}}{z_{1}^{2}}\right|$ est borné. Écrivons $A^{\prime}=A_{1}^{\prime}+A_{2}^{\prime}$, où $A_{1}^{\prime}$ est la partie linéaire de $A^{\prime}$. Par la condition (2) sur $\omega_{\text {lin }}$, les valeurs propres $\lambda$ et $\mu$ sont différents de ), donc $\left|\frac{A_{1}^{\prime}}{z_{1}}\right| \geq l$ pour $l \in \mathbb{R}$ positif. Comme $\left|\frac{A_{2}^{\prime}}{z_{1}}\right|$ est petit pour $\left|\left(z_{1}, z_{2}\right)\right|$ petit, on a l'estimation.

Par conséquent, le champ $\Sigma^{\mathbb{C}}(\mathcal{F})$ (qui est analytique réel, sans singularités hors de $\left.\left\{z_{1}=0\right\}\right)$ complété par $\left\{z_{1}=0\right\}$ devient continu ; on peut dire plus. Les feuilles de $\mathcal{F}^{\mathbb{C}}$ sont tranverses aux sections $z_{2}=$ cste ; alors, pour chaque $m \in \mathbb{C}$ petit on peut définir l'application d'holonomie $H^{m}:\left\{z_{2}=0\right\} \rightarrow\left\{z_{2}=m\right\}$ le long de $\left\{z_{1}=0\right\}\left(H^{m}\left(z_{1}, 0\right)=\left(z_{1}^{\prime}, m\right)\right.$ lorsque $\left(z_{1}, 0\right)$ et $\left(z_{1}^{\prime}, m\right)$ appartiennent à la même feuille de $\mathcal{F}^{\mathbb{C}}$, et $\left.H^{m}(0,0)=(0, m)\right)$. L'inégalité donnée dans l'affirmation précédente nous permet d'appliquer le théorème de dépendance des solutions d'une équation différentielle par raport aux conditions initiales à l'équation $\Omega=0$ (avec $z_{2}=t m, t \in \mathbb{R}$ ) pour déduire que $H^{m}$ est continue. Evidemment l'application correspondante pour $\mathcal{F}_{\text {lin }}^{\mathbb{C}}$ est $H_{\text {lin }}^{m}\left(z_{1}, 0\right)=\left(z_{1}, m\right)$.

Les feuilletages $\mathcal{F}$ et $\mathcal{F}_{\text {lin }}$ coupent la droite $\left\{z_{2}=0\right\}$ selon deux feuilletages en courbes, singuliers à l'origine ; par les conditions (2) et (3), ils sont analytiquement conjugués par un difféomorphisme holomorphe $\phi_{0}$ (Théorème de Poincaré, [Po]). Pour finir la preuve, il suffit d'étendre $\phi_{0}$ à chaque section $\left\{z_{2}=m\right\}$ comme

$$
\phi_{m}\left(z_{1}, m\right)=H_{\operatorname{lin}}^{m} \circ \phi_{0} \circ\left(H^{m}\right)^{-1}\left(z_{1}, m\right) .
$$


REMARQue. Le feuilletage en courbes complexes associé au feuilletage Levi-flat défini par $\mathcal{R} e\left[z_{1}+t\left(i z_{1}+z_{1}^{m} z_{2}\right)\right]=0$, où $t \in R$ et $m \geq 1$, est seulement de classe $C^{m-1}$ le long de $\left\{z_{1}=0\right\}$ (l'application d'holonomie le long de cette feuille est de classe $C^{m-1}$ ).

\section{7. - Fonctions de Morse Levi-flat}

Le but de cette section est d'étudier les 1-formes différentielles intégrables Levi-flat dont la partie linéaire est donné par (5.1) avec $k=n$. Selon un théorème de R. Moussu [Mo], une 1-forme différentielle analytique intégrable $\eta$ à singularité simple (ou de Morse) en $0 \in \mathbb{R}^{m}(m \geq 3)$,

$$
\eta=d\left(\sum_{j=1}^{k} x_{j}^{2}-\sum_{j=k+1}^{m} x_{j}^{2}\right)+\ldots
$$

possède une intégrale première analytique du type

$$
f=\sum_{j=1}^{k} x_{j}^{2}-\sum_{j=k+1}^{m} x_{j}^{2}+\cdots
$$

Notre problème est d'étudier une telle fonction dans une voisinage de $0 \in \mathbb{C}^{n}$ lorsque le feuilletage associé est Levi-flat. Soient alors $\mathcal{F}_{\text {lin }}$ et $\mathcal{F}$ les feuilletages définis par les niveaux de $f_{0}=\mathcal{R} e\left(\sum_{j=1}^{n} z_{j}^{2}\right)$ et de $f=f_{0}+\ldots$ respectivement. Le résultat de Burns et Gong, voir [B-G], mentionné dans l'introduction, nous permet de supposer que les niveaux $f=0$ et $f_{0}=0$ sont les mêmes ; par conséquent, $f=V . f_{0}$, pour une fonction analytique réelle $V$ avec $V(0)=1$. On note $S$ la quadrique complexe $\left\{\sum_{j=1}^{n} z_{j}^{2}=0\right\}$.

L'idée maintenant est de faire une explosion à l'origine de $\mathbb{C}^{n}$ et d'analyser les feuilletages éclatés correspondants $\hat{\mathcal{F}}_{\text {lin }}^{\mathbb{C}}$ et $\hat{\mathcal{F}}^{\mathbb{C}}$. Leurs équations respectives sont (dans le système de coordonnées où $z_{j}=t_{j} z_{1}, 2 \leq j \leq n$ ) :

$$
l d z_{1}+z_{1} \sum_{j=2}^{n} t_{j} d t_{j}=0
$$

et

$$
\left(z_{1} l+q \beta\right) d z_{1}+\sum_{j=2}^{n}\left(z_{1}^{2} t_{j}+z_{1} q \alpha_{j}\right) d t_{j}=0
$$

avec $\mathbf{t}=\left(t_{2}, \ldots, t_{n}\right), l=l(\mathbf{t})=\left(1+t_{2}^{2}+\cdots+t_{n}^{2}\right)$ et $q\left(z_{1}, \mathbf{t}\right)=\mathcal{R} e z_{1}^{2} l(\mathbf{t})$; les fonctions $\alpha_{j}$ et $\beta$ sont analytiques réelles. Le diviseur exceptionnel $E$ est invariant par $\hat{\mathcal{F}}_{\text {lin }}^{\mathbb{C}}$, dont l'ensemble singulier est $E \cap \hat{S}$ ( $\hat{S}$ est le transformé strict de $S$ ) 
Lemme 7.1. Le feuilletage $\hat{\mathcal{F}}^{\mathbb{C}}$ est de classe $C^{2}$ le long de $E^{\prime}=E \backslash \hat{S}$ et de classe $C^{1}$ sur $E \cap \hat{S}$.

Preuve. Regardons l'équation (7.2) sous la forme

$$
d z_{1}+\sum_{j=2}^{n}\left(\frac{z_{1} t_{j}+q \alpha_{j}}{l+z^{-1} q \beta}\right) d t_{j}=0
$$

si $l(\mathbf{t}) \neq 0$; on constate facilement que les fonctions $\frac{z_{1} t_{j}+q \alpha_{j}}{l+z_{1}^{-1} q \beta}$ (définies comme 0 au point $(0, \mathbf{t}))$ sont de classe $C^{2}$. Par contre, dans un voisinage de $\mathbf{b} \in E$ tel que $l(\mathbf{b})=0$, il faut considérer (7.2) sous la forme

$$
\left(l+z_{1}^{-1} q \beta\right) d z_{1}+\sum_{j=2}^{n}\left(z_{1} t_{j}+q \alpha_{j}\right) d t_{j}=0
$$

qui est de classe $C^{1}$ (la fonction $z_{1}^{-1} q \beta$ est définie comme 0 au point $(0, \mathbf{b})$ ).

Comme dans le cas linéaire, le diviseur exceptionnel $E$ est invariant et $E \cap \hat{S}$ est l'ensemble singulier. De plus, $E \cap \hat{S}$ est un ensemble singulier de type Reeb-Kupka pour les deux feuilletages.

Si $n \geq 3$, d'après Zariski le groupe fondamental de $E^{\prime}:=E \backslash \hat{S}$ est isomorphe a $\mathbb{Z}_{2}$, en particulier les groupes d'holonomie $\mathcal{H o l}\left(\hat{\mathcal{F}}_{\text {lin }}^{\mathbb{C}}, E^{\prime}\right)$ et $\mathcal{H o l}\left(\hat{\mathcal{F}}^{\mathbb{C}}, E^{\prime}\right)$ sont engendrés respectivement par $\gamma_{\text {lin }}$ et $\gamma$ qui sont conjugués à l'involution linéaire $z \mapsto-z$. Par exemple, les feuilles de $\mathcal{F}_{\text {lin }}^{\mathbb{C}}$ sont données par $\sum_{j=1}^{n} z_{j}^{2}=c$ pour $c \in \mathbb{C}$; si $z_{j}=t_{j} z_{1}, j=2, \ldots n$, alors chaque feuille de $\hat{\mathcal{F}}_{\text {lin }}^{\mathbb{C}}$ coupe la section transversale $\Gamma_{\mathbf{a}}:\{\mathbf{t}=\mathbf{a}\}$ à $E^{\prime}$ selon l'ensemble défini par l'equation $z_{1}^{2}=\left(1+\sum_{j=2}^{n} a_{j}^{2}\right)^{-1} c$, et ainsi $\gamma_{\text {lin }}\left(z_{1}\right)=-z_{1}$. Nous allons trouver une conjugaison entre $\gamma_{\text {lin }}$ et $\gamma$ respectant les traces de $\hat{\mathcal{F}}_{\text {lin }}$ et de $\hat{\mathcal{F}}$ sur la section $\Gamma_{\mathbf{a}}$; ensuite, cette conjugaison sera poussée le long des feuilles de $\hat{\mathcal{F}}_{\text {lin }}^{\mathbb{C}}$ et $\hat{\mathcal{F}}^{\mathbb{C}}$.

Soient $H^{\mathbf{a}, \mathbf{t}}$ et $H_{\text {lin }}^{\text {a,t }}$ les applications d'holonomie de $E^{\prime}$ entre les sections $\Gamma_{\mathbf{a}}$ et $\Gamma_{\mathbf{t}}, \mathbf{a}, \mathbf{t} \in E^{\prime}$ pour les feuilletages $\hat{\mathcal{F}}_{\text {lin }}^{\mathbb{C}}$ et $\hat{\mathcal{F}}^{\mathbb{C}}$. Évidemment $H_{\text {lin }}^{\mathbf{a}, \mathbf{t}}$ est holomorphe, et $H^{\mathrm{a}, \mathrm{t}}$ est une application de classe $C^{2}$ d'après le Lemme 7.1 (analytique réelle hors de $E$ ).

COROLlary 7.1. Le génerateur $\gamma$ est une application de classe $C^{2}$ (analytique réelle en dehors de $\left.0 \in \Gamma_{\mathbf{a}}\right), C^{2}$-conjuguée à l'involution $\gamma_{\operatorname{lin}}\left(z_{1}\right)=-z_{1}$ par $\phi=$ $\frac{1}{2}(I d-\gamma)$.

On considère maintenant les restrictions à $\Gamma_{\mathbf{a}}$ de $f_{0}\left(z_{1}, t_{2} z_{1}, \ldots, t_{n} z_{1}\right)$ et $f\left(z_{1}, t_{2} z_{1}, \ldots, t_{n} z_{1}\right)$, que l'on note $q\left(z_{1}\right)$ et $q\left(z_{1}\right)+h\left(z_{1}\right)$, où le 2 -jet de $h$ est zéro.

Lemme 7.2. Il existe un difféomorphisme local $\psi$ de $\Gamma_{\mathbf{a}} \simeq \mathbb{C}, 0$ tel que $d \psi(0)=$ $I d,(q+h) \circ \psi=q$ et $\psi \circ \gamma=\gamma_{\text {lin }} \circ \psi ; \psi$ est analytique réel en dehors de $0 \in \mathbb{C}$ et $C^{1}$ en $0 \in \mathbb{C}$. 
Preuve. Il s'agit du lemme de Morse équivariant ; la démonstration se fait par la méthode du chemin. Après avoir conjugué $\gamma$ par $\phi$, on peut supposer que $(q+h) \circ \gamma_{\text {lin }}=q+h$; on cherche $\psi$ satisfaisant le lemme tel que $\psi \circ \gamma_{\text {lin }}=\gamma_{\operatorname{lin}} \circ \psi$ (notons qu'après conjugaison par $\phi$, la nouvelle fonction $q+h$ est de classe $C^{2}$, analytique réelle en dehors de $0 \in \Gamma_{\mathbf{a}}$ ).

La démonstration du lemme de Morse ordinaire procède de la façon suivante. On considère la fonction

$$
F\left(s, z_{1}\right)=q\left(z_{1}\right)+\operatorname{sh}\left(z_{1}\right)
$$

sur un petit voisinage de $[0,1] \times\{0\}$ dans $\mathbb{C} \times \mathbb{C}$; on construit un champ de vecteurs

$$
Y=a\left(s, z_{1}\right) \frac{\delta}{\delta x_{1}}+b\left(s, z_{1}\right) \frac{\delta}{\delta x_{2}}+\frac{\delta}{\delta s}, a\left(0, z_{1}\right)=b\left(0, z_{1}\right)=0 .
$$

tangent au feuilletage $F=$ cste, i.e., $Y(F)=0$.

On conclut que, si $\phi_{t}\left(s, z_{1}\right)$ est le flot du champ $Y$,

$$
\phi_{t}\left(s, z_{1}\right)=\left(s+t, \psi_{t}\left(s, z_{1}\right)\right),
$$

alors le difféomorphisme $\xi: z_{1} \mapsto \phi_{1}\left(0, z_{1}\right)$ conjugue $F_{0}=q$ et $F_{1}=q+h$. On modifie la preuve de la façon suivante ; on considère l'involution $\sigma\left(s, z_{1}\right)=$ $\left(s,-z_{1}\right)$. Le champ $X=\frac{1}{2}\left(Y+\sigma_{*} Y\right)$ est invariant par $\sigma$ et tangent au feuilletage $F=$ cste. La conclusion précédente cette fois avec $X$ nous donne un difféomorphisme $\xi$ tel que $(q+h) \circ \xi=q$. Comme $q \circ \xi^{\prime}(0)=q$, on peut prendre finalement $\psi=\xi^{\prime}(0) \circ \xi^{-1}$.

Maintenant le difféomorphisme $\psi$ défini sur $\Gamma_{\mathbf{a}}$ doit être poussé le long des feuilles de $\hat{\mathcal{F}}_{\text {lin }}^{\mathbb{C}}$ et $\hat{\mathcal{F}}^{\mathbb{C}}$ en respectant la fibration de Hopf ; le Lemme 7.2 garantit que ce prolongement est bien défini et donne aussi une conjugaison entre $\hat{\mathcal{F}}_{\text {lin }}$ et $\hat{\mathcal{F}}$. Il reste à analyser la nature de cette extension sur $\hat{S}$.

THÉORÈme 7.1. Si $n \geq 3$, il existe une conjugaison continue entre $\mathcal{F}_{\text {lin }}$ et $\mathcal{F}$, $C R$ en dehors de $S$.

Preuve. Soit $\psi_{\mathbf{t}}: \Gamma_{\mathbf{t}} \rightarrow \Gamma_{\mathbf{t}}=H^{\mathbf{a}, \mathbf{t}} \circ \psi \circ H_{\text {lin }}^{\mathbf{t}, \mathbf{a}}$ le prolongement défini ci-dessus. Nous allons montrer que si $\mathbf{t} \rightarrow \mathbf{b} \in E \cap \hat{S}$ alors $\psi_{\mathbf{t}} \rightarrow I d$. Comme $E \cap \hat{S}$ est de type Kupka-Reeb, on peut supposer que $\mathbf{t} \in E \backslash \hat{S}$ appartient à une section transversale (de dimension complexe 1) à $E \cap \hat{S}$ par le point $\mathbf{b}$, disons $t_{j}=b_{j}, j \geq 3$ et $b_{2} \neq 0$. Fixons $\mathbf{a} \in E^{\prime}$ dans la même section ; on a $\left(H_{\text {lin }}^{\mathbf{t}, \mathbf{a}}\left(z_{1}\right)\right)^{2}=\frac{l(\mathbf{t})}{l(\mathbf{a})} z_{1}^{2}$. Écrivons (7.1) et (7.2) comme $\left(u=t_{2}-b_{2}\right)$ :

$$
\left(2 b_{2} u+u^{2}\right) d z_{1}+\left(b_{2} z_{1}+z_{1} u\right) d u=0
$$


et

$$
\left(2 b_{2} u+u^{2}+z_{1}^{-1} q \beta\right) d z_{1}+\left(b_{2} z_{1}+z_{1} u+q \alpha\right) d u=0
$$

D'après le Lemme 7.1 , ces équations sont de classe $C^{1}$; on voit que les parties linéaires sont les mêmes. Ainsi, pour $\left|z_{1}\right|<r$,

$$
\lim _{\mathbf{t} \rightarrow \mathbf{b}} H^{\mathbf{a}, \mathbf{t}} \circ H_{\operatorname{lin}}^{\mathbf{t}, \mathbf{a}}=I d .
$$

Comme

$$
\lim _{\mathbf{t} \rightarrow \mathbf{b}} H_{\operatorname{lin}}^{\mathbf{a}, \mathbf{t}} \circ \psi \circ H_{\text {lin }}^{\mathbf{t}, \mathbf{a}}=d \psi(0)=I d
$$

uniformément dans $\left|z_{1}\right|<r$, on peut conclure que

$$
\lim _{\mathbf{t} \rightarrow \mathbf{b}} \psi_{\mathbf{t}}=\lim _{\mathbf{t} \rightarrow \mathbf{b}} H^{\mathbf{a}, \mathbf{t}} \circ H_{\operatorname{lin}}^{\mathbf{t}, \mathbf{a}} \circ H_{\operatorname{lin}}^{\mathbf{a}, \mathbf{t}} \circ \psi \circ H_{\operatorname{lin}}^{\mathbf{t}, \mathbf{a}}=I d .
$$

Remarque. Si $n=2, E \simeq \mathbb{C P}(1)$ et l'intersection $E \cap \hat{S}$ est réduite à 2 points. Donc le groupe fondamental de $E^{\prime}$ est isomorphe à $\mathbb{Z}$, et on ne peut pas directement conclure que les feuilles complexes sont fermées dans un voisinage du diviseur exceptionnel.

\section{8. - Phénomène de Kupka-Reeb avec ensemble singulier non-holomorphe}

Dans cette section on étudie les perturbations Levi-flat du cas linéaire (5.2).

Notons d'abord que nous pouvons supposer que $\operatorname{Sing} \mathcal{F}=\left\{x_{1}=x_{2}=0\right\}$. En fait, notons qu'en chaque point du lieu singulier $\operatorname{Sing} \mathcal{F}$ - qui est une variété lisse de codimension 2 réelle- le 1-jet est de même type. En particulier, en chaque point $m \in \operatorname{Sing} \mathcal{F}$, il existe un $\mathbb{C}$ - espace vectoriel $\mathcal{T}_{m}$ de codimension 2 contenu dans $T_{m} \operatorname{Sing} \mathcal{F}$. Le théorème de linéarisation de Poincaré [Po] et le théorème de Kupka $[\mathrm{K}]$, cf. Section 6, nous assurent l'existence de deux submersions $f$ et $g$ analytiques réelles telles que $f d g-g d f$ définit $\mathcal{F}$. Le lieu singulier Sing $\mathcal{F}$ apparait comme l'intersection des hypersurfaces Levi-flat lisses et transverses $(f=0)$ et $(g=0)$. En résulte que si $m \in \operatorname{Sing} \mathcal{F}$ alors $\mathcal{T}_{m}$ est exactement $T_{m}^{\mathbb{C}}(f=0) \cap T_{m}^{\mathbb{C}}(g=0)$; ceci implique que le champ $\mathcal{T}_{m}$ est donné par un système intégrable sur $\operatorname{Sing} \mathcal{F}$ : l'intersection des restrictions à Sing $\mathcal{F}$ des systèmes intégrables $\left.T_{m}^{\mathbb{C}}(f=0)\right|_{\text {Sing } \mathcal{F}}$ et $\left.T_{m}^{\mathbb{C}}(g=0)\right|_{\text {Sing } \mathcal{F}}$. La feuille passant par $m$ est une variété complexe lisse contenue dans $\operatorname{Sing} \mathcal{F}$. La feuille de $\mathcal{T}$ passant par le point $\left(z_{1}=i y_{1}, z_{2}=i y_{2}, z_{2}=\ldots z_{n}=0\right)$ est paramétrée par une application du type

$$
z^{\prime}=\left(z_{3}, \ldots, z_{n}\right) \mapsto\left(\phi_{1}\left(i y_{1}, i y_{2}, z^{\prime}\right), \phi_{2}\left(i y_{1}, i y_{2}, z^{\prime}\right), z^{\prime}\right)
$$


holomorphe en $z^{\prime}$ et analytique réelle en $y_{1}, y_{2}$. Considérons l'unique application holomorphe $\tilde{\phi}$ :

$$
\left.\left(z_{1}, z_{2}, z^{\prime}\right) \mapsto\left(\tilde{\phi}_{1}\left(z_{1}, z_{2}, z^{\prime}\right), z^{\prime}\right), \tilde{\phi}_{2}\left(z_{1}, z_{2}, z^{\prime}\right), z^{\prime}\right)
$$

telle que $\tilde{\phi}_{j}\left(i y_{1}, i y_{2}, z^{\prime}\right)=\phi_{j}\left(i y_{1}, i y_{2}, z^{\prime}\right), j=1,2$. Visiblement $\tilde{\phi}$ est un difféomorphisme local et l'image par $\tilde{\phi}$ de $\left(x_{1}=x_{2}=0\right)$ est précisément l'ensemble $\operatorname{Sing} \mathcal{F}$. De sorte que dans la suite nous pouvons supposer que Sing $\mathcal{F}=\left\{x_{1}=x_{2}=0\right\}$.

Considérons l'adhérence d'une feuille de $\mathcal{F}$; elle est donnée par $f / g=t$, où $t \in \mathbb{R} \mathbb{P}(1)$. Elle est d'autre part lisse et Levi-flat, donc feuilletée par des sous-variétés holomorphes $\mathcal{L}_{t, s}$ de codimension 1. Les intersections $\mathcal{G}_{t, s}=\mathcal{L}_{t, s} \cap$ $\operatorname{Sing} \mathcal{F}$ produisent un feuilletage analytique réel $\mathcal{G}_{t}$ régulier de $\operatorname{Sing} \mathcal{F}=\left\{x_{1}=\right.$ $\left.x_{2}=0\right\}$; on note que chaque $\mathcal{G}_{t, s}$ contient un ouvert d'un $(n-2)$-espace affine $\left\{\left(i y_{1}, i y_{2}, z^{\prime}\right) ; z^{\prime} \in \mathbb{C}^{n-2}\right)$. On construit ainsi une famille $\mathcal{G}$ de feuilletages $\mathcal{G}_{t}, t \in \mathbb{R} \mathbb{P}(1)$, sur l'espace $\operatorname{Sing} \mathcal{F}$. Noter, comme on l'a déjà dit, que cette famille est trivialisée par le feuilletage complexe engendré par les champs de vecteurs $\partial / \partial z_{3}, \ldots, \partial / \partial z_{n}$. Finalement la donnée de la famille $\mathcal{G}$ revient à celle de la restriction $\mathcal{G}_{0}=\mathcal{G} \mid\left(z^{\prime}=0\right)$; on note que $\mathcal{G}_{0}$ est infinitésimalement donné par la famille de droites parallèles correspondant à l'exemple linéaire $x_{1} d x_{2}-x_{2} d x_{1}=0$.

DÉFINITION 8.1. On appelle famille régulière $\mathcal{H}$ de feuilletages en l'origine de $\mathbb{R}^{2}$ la donnée de feuilletages réguliers analytiques $\mathcal{H}_{t}, t \in \mathbb{R} \mathbb{P}(1)$, la tangente $T_{t}$ à la feuille de $\mathcal{H}_{t}$ par $0 \in \mathbb{R}^{2}$ correspondant au paramètre $t: T_{t}=\left\{y_{2}=t y_{1}\right\}$; la dépendance de $\mathcal{H}_{t}$ doit être analytique par rapport à $t \in \mathbb{R} \mathbb{P}(1)$.

Ainsi à un feuilletage $\mathcal{F}$ comme ci-dessus on associe une famille régulière de feuilletages du plan $\mathbb{R}^{2}$ : le feuilletage $\mathcal{G}_{0}$ obtenu par la construction précédente.

Deux familles régulières $\mathcal{H}$ et $\mathcal{H}^{\prime}$ de feuilletages en $0 \in \mathbb{R}^{2}$ sont conjuguées s'il existe un difféomorphisme local $\phi$ analytique en 0 tel que $\phi\left(\mathcal{H}_{t}\right)=\mathcal{H}_{t}^{\prime}$.

La preuve du théorème suivant $\mathrm{a}$ un corollaire important :

THÉORÈME 8.2. Soient $\mathcal{F}$ et $\mathcal{F}^{\prime}$ deux feuilletages analytiques réels Levi-flat à l'origine de $\mathbb{C}^{n}$. On suppose que $\mathcal{F}$ et $\mathcal{F}^{\prime}$ sont donnés par les 1 -formes $\omega$ et $\omega^{\prime}$ avec $j^{1} \omega=j^{1} \omega^{\prime}=x_{1} d x_{2}-x_{2} d x_{1}$. Alors $\mathcal{F}$ et $\mathcal{F}^{\prime}$ sont $C R$ - conjugués ${ }^{(1)}$ si et seulement si les familles régulières associées $\mathcal{G}_{0}$ et $\mathcal{G}_{0}^{\prime}$ sont conjuguées. De plus, si $\mathcal{G}_{0}^{\prime \prime}$ est une famille régulière de feuilletages en l'origine de $\mathbb{R}^{2}$, il existe un feuilletage $\mathcal{F}^{\prime \prime}$ Levi-flat comme ci-dessus ayant pour famille associée $\mathcal{G}_{0}^{\prime \prime}$.

Preuve. On reprend les notations précédentes. On peut supposer d'abord que Sing $\mathcal{F}=\operatorname{Sing} \mathcal{F}^{\prime}=\left\{x_{1}=x_{2}=0\right\}$. On remarque ensuite que si $\phi$ est un difféomorphisme conjugant $\mathcal{F}$ à $\mathcal{F}^{\prime}$ alors $\phi$ laisse invariant $\left\{x_{1}=x_{2}=0\right\}$. Si $\phi$

${ }^{(1)}$ Par un difféomorphisme analytique qui est CR en dehors du lieu $\operatorname{singulier} \operatorname{Sing}(\mathcal{F})$. 
est de plus CR, l'image de la feuille holomorphe $\mathcal{L}_{t, s}$ introduite précédemment est une feuille correspondante $\mathcal{L}_{t^{\prime}, s^{\prime}}$. Comme $\phi$ préserve $\left\{x_{1}=x_{2}=0\right\}$ on a

$$
\phi\left(\mathcal{L}_{t, s} \cap \operatorname{Sing} \mathcal{F}\right)=\mathcal{L}_{t^{\prime}, s^{\prime}}^{\prime} \cap \operatorname{Sing} \mathcal{F}^{\prime} .
$$

Remarquons que $\phi$ préserve le feuilletage de $\operatorname{Sing} \mathcal{F}$ donné par les champs de vecteurs $\partial / \partial z_{3}, \ldots, \partial / \partial z_{n}$. Par conséquent, $\phi$ induit une conjugaison entre $\mathcal{G}_{0}$ et $\mathcal{G}_{0}^{\prime}$.

Supposons maintenant que $\phi:\left(i y_{1}, i y_{2}\right) \mapsto \phi\left(i y_{1}, i y_{2}\right)$ conjugue $\mathcal{G}_{0}$ et $\mathcal{G}_{0}^{\prime}$. Soit $\psi: \mathbb{C}^{n} \rightarrow \mathbb{C}^{n}$ définit par

$$
\left(z_{1}, z_{2}, z_{3}, \ldots, z_{n}\right) \mapsto\left(\tilde{\phi}\left(z_{1}, z_{2}\right), z_{3}, \ldots z_{n}\right)
$$

où $\tilde{\phi}$ est l'extension holomorphe de $\phi$. Considérons le feuilletage analytique réel Levi-flat $\tilde{\mathcal{F}}=\psi(\mathcal{F})$; avec les notations précédents $\tilde{\mathcal{L}}_{t, s}=\psi\left(\mathcal{L}_{t, s}\right)$ et $\psi\left(\mathcal{L}_{t, s}\right) \cap\left\{x_{1}=x_{2}=0\right\}=\tilde{\mathcal{L}}_{t, s} \cap\left\{x_{1}=x_{2}=0\right\}$. Mais par construction $\tilde{\mathcal{L}}_{t, s} \cap\left\{x_{1}=x_{2}=0\right\}=\mathcal{L}_{t^{\prime}, s^{\prime}}^{\prime} \cap\left\{x_{1}=x_{2}=0\right\}$; les hypersurfaces holomorphes $\tilde{\mathcal{L}}_{t, s}$ et $\mathcal{L}_{t^{\prime}, s^{\prime}}^{\prime}$ contiennent donc un ensemble analytique réel de codimension un, donc sont égales. On en déduit facilement que $\psi(\mathcal{F})=\mathcal{F}^{\prime}$.

Donnons nous maintenant une famille régulière $\mathcal{G}^{\prime \prime}$ à l'origine de $\mathbb{R}^{2}=$ $\left\{\left(y_{1}, y_{2}\right)\right\}$; le feuilletage $\mathcal{G}_{0, t}^{\prime \prime}$ est donné par

$$
\left\{\left(y_{1}, y_{2}\right) \in \mathbb{R}^{2} ; \phi\left(i y_{1}, i y_{2}, t, s\right)=0\right\}
$$

où $\phi$ est analytique submersive en $(0,0, t, 0)$ et $\operatorname{Ker} d_{(0,0, t, 0)} \phi=[t]$. Considérons le feuilletage analytique réel en hypersurfaces holomorphes de $\mathbb{C}^{n}$ dont les feuilles paramétrées par $(t, s)$ sont données par

$$
\mathcal{L}_{t, s}^{\prime \prime}=\left\{\left(z_{1}, z_{2}, \ldots, z_{n}\right) ; \tilde{\phi}\left(z_{1}, z_{2}, t, s\right)=0\right\}
$$

où $\tilde{\phi}$ est l'extension holomorphe de $\phi$. On définit alors le feuilletage analytique réel $\mathcal{F}^{\prime \prime}$ dont les feuilles sont les $\cup_{s} \mathcal{L}_{t, s}^{\prime \prime}$. Ce feuilletage qui a pour lieu singulier $\left\{x_{1}=x_{2}=0\right\}$ convient.

Une conséquence de la preuve est la suivante :

COROLlaire 8.1. Si $\mathcal{F}$ et $\mathcal{F}^{\prime}$ comme précédemment sont CR-conjugués, ils le sont holomorphiquement.

On peut comprendre ce corollaire en revenant à l'exemple linéaire $x_{1} d x_{2}-$ $x_{2} d x_{1}=0$ dans $\mathbb{C}^{2}$. Le feuilletage en courbes holomorphes sous jacent est donné par le champ de vecteurs $x_{1} \partial / \partial z_{1}+x_{2} \partial / \partial z_{2}$, dont les feuilles sont données par $z_{2}-t z_{1}=i s$; soient $U$ un voisinage de $0 \in \mathbb{C}^{2}$ et $f: U \rightarrow \mathbb{C}$ une fonction analytique réelle. On suppose que $f$ est holomorphe en restriction à chaque 
droite $\left\{z_{2}-t z_{1}=i s\right\} \cap U$; alors $f$ est holomorphe. En effet, la condition implique que

$$
\left(x_{1} \partial / \partial z_{1}+x_{2} \partial / \partial z_{2}\right)(\bar{f})=0 .
$$

Si on développe $f$ comme $f=\sum_{k, l} \bar{a}_{k l}\left(z_{1}, z_{2}\right) z_{1}^{k} z_{2}^{l}$, où les $a_{k l}$ sont holomorphes, on a

$$
\begin{gathered}
\sum_{k, l}\left(x_{1} \frac{\partial}{\partial z_{1}}+x_{2} \frac{\partial}{\partial z_{2}}\right)\left(a_{k l}\right) \bar{z}_{1}^{k} \bar{z}_{2}^{l}= \\
\sum_{k, l}\left(z_{1} \frac{\partial}{\partial z_{1}}+z_{2} \frac{\partial}{\partial z_{2}}\right)\left(a_{k l}\right) \bar{z}_{1}^{k} \bar{z}_{2}^{l}+\sum_{k, l} \frac{\partial a_{k l}}{\partial z_{1}} \bar{z}_{1}^{k+1} z_{2}^{l}+\sum_{k l} \frac{\partial a_{k l}}{\partial z_{2}} \bar{z}_{1}^{k} z_{2}^{l+1}=0 .
\end{gathered}
$$

Supposons que l'on ait montré que les fonctions $a_{k l}$ sont constantes pour $k+l<$ $m$. On obtient pour $k+l=m$ que

$$
\sum_{k, l}\left(z_{1} \frac{\partial}{\partial z_{1}}+z_{2} \frac{\partial}{\partial z_{2}}\right)\left(a_{k l}\right) \bar{z}_{1}^{k} \bar{z}_{2}^{l}=0,
$$

qui implique que $a_{k l}$ est constant.

\section{REFERENCES}

[Ca] E. CARTAN, Sur la géométrie pseudo-conforme des hypersurfaces de l'espace de deux variables complexes, Ann. Mat. Pura Appl. (4), 11 (1933), 17-90.

[C-M] S.S. CHERN - J. MOSER, Real hypersurfaces in complex manifolds, Acta Math. 133 (1974), 219-271.

[Ba] M.S. Baouend - P. Ebenfelt - L. Rothschild, "Real submanifolds in complex space and their mappings", Princeton Mathematical Series, 47 (1999).

[B-G] D. BuRns - X. Gong, Singular Levi-flat real analytic hypersurfaces, Amer. J. Math. 121 (1999), 23-53.

[K] I. KUPKA, The singularities of integrable structurally stable Pfaffian forms, Proc. Natl. Acad. Sci. USA 52 (1964), 1431-1432.

[Mal I] B. Malgrange, Frobenius avec singularités I : Codimension un, Inst. Hautes Études Sci. Publ. Math. No. 46 (1976), 163-173.

[Mal II] B. Malgrange, Frobenius avec singularités II : Le cas général, Invent. Math. 39 (1977), 67-89.

[Mo] R. Moussu, Sur l'existence d'intégrales premières pour un germe de forme de Pfaff, Ann. Inst. Fourier (Grenoble) 26 (1976), 171-220.

[Po] H. Poincaré, "Note sur les propriétés des fonctions définies par les équations différentielles”, Oeuvres t.I, XXXVI, Gauthier-Villars, 1951. 
[Re] G. Reeb, "Sur certains propriétés topologiques des variétés feuilletés", Actual. Sci. Indust., 1183, Hermann, 1952.

[Sa] K. SAIto, On a generalization of de-Rham lemma, Ann. Inst. Fourier (Grenoble) 26 (1976), 165-170.

Institut de Recherches Mathématiques de Rennes

Université de Rennes 1

Campus de Beaulieu

35042 Rennes, France

dominique.cerveau@univ-rennes.fr

Instituto de Matemática Pura e Aplicada

Estrada D. Castorina 110, Jardim Botânico

22320-010 Rio de Janeiro, Brasil

sad@impa.br 